\title{
Revisiting three minerals from Cioclovina Cave (Romania)
}

\author{
Bogdan P. Onac ${ }^{1,2^{*}}$, Herta S. Effenberger ${ }^{3}$, Nathan C. Collins ${ }^{1}$, Joe B. Kearns ${ }^{4}$, and Radu
} C. Breban ${ }^{5}$

\begin{abstract}
:
Onac B.P., Effenberger H.S., Collins N.C., Kearns J.B. and Breban R.C., 2011. Revisiting three minerals from Cioclovina Cave (Romania). International Journal of Speleology, 40(2), 99-108. ISSN 0392-6672. DOI 10.5038/1827-806X.40.2.2

Cioclovina Cave in Romania's Southern Carpathians is a world-renowned cave site for its paleontological, anthropological, and mineralogical (type locality of ardealite) finds. To date, over 25 mineral species have been documented, some unusual for a cave environment. This paper presents details on the occurrence of collinsite $\left[\mathrm{Ca}_{2}\left(\mathrm{Mg}, \mathrm{Fe}^{2+}\right)\left(\mathrm{PO}_{4}\right)_{2} \cdot 2 \mathrm{H}_{2} \mathrm{O}\right]$, atacamite $\left[\mathrm{Cu}{ }_{2}{ }^{2+} \mathrm{Cl}(\mathrm{OH})_{3}\right]$, and kröhnkite $\left[\mathrm{Na}_{2} \mathrm{Cu}^{2+}\left(\mathrm{SO}_{4}\right)_{2} \cdot 2 \mathrm{H}_{2} \mathrm{O}\right]$ based on single-crystal X-ray diffraction, electron microprobe, stable isotope analyses, and scanning electron microscope imaging. This is the first reported occurrence of kröhnkite in a cave environment. Atacamite represents the weathering product (in the presence of Lower-Cretaceous limestone-derived chlorine) of copper minerals washed into the cave from nearby ore bodies. Atacamite and kröhnkite have similar sources for copper and chlorine, whereas sodium probably originates from weathered Precambrian and Permian detrital rocks. Collinsite is believed to have precipitated from bat guano in a damp, nearneutral $\mathrm{pH}$ environment. The results show the following sequence of precipitation: ardealite-brushite-(gypsum)-atacamite-kröhnkite. This suggests that the observed mineral paragenesis is controlled by the neutralization potential of the host-rock mineralogy and the concentrations of $\mathrm{Ca}, \mathrm{Cl}, \mathrm{Cu}$, and $\mathrm{Na}$.
\end{abstract}

Keywords: cave minerals, sulfates, phosphates, guano, stable isotopes, Cioclovina Cave, Romania

Received 18 December 2010; Revised 20 January 2011; Accepted 5 February 2011

\section{INTRODUCTION}

The investigated area is in the Grădiştea Muncelului-Cioclovina Natural Park, located in the western part of the Şureanu Mountains (also known as Sebeş Mts.), a major unit of the Transylvanian Alps (Martonne, 1907; Fig. 1a). Cioclovina Cave is a world-renowned site for its paleontological (Simionescu, 1941; Breban et al., 2003), anthropological (Olariu et al., 2002; Soficaru et al., 2007; Alexandrescu et al., 2010), and mineralogical (Onac et al., 2002, 2007; Onac \& Effenberger, 2007; Dumitraş, 2008; Onac \& Tămaş, 2010) finds, being declared a scientific reserve since 1979 . The cave represents the type locality of ardealite (Schadler, 1932). From a total of $1,400 \mathrm{~m}$ of surveyed passages (Tomuş, 1999;

${ }^{1}$ Department of Geology, University of South Florida, 4202 E. Fowler Ave., Tampa, FL 33620, USA (*bonac@usf.edu)

${ }^{2}$ Department of Geology, "Babeş-Bolyai" University/"Emil Racoviță” Institute of Speleology, Cluj, Kogălniceanu 1, 400084 Cluj, Romania

${ }^{3}$ Institute of Mineralogy and Crystallography, University of Wien, Geozentrum, Althanstraße 14, A-1090 Austria

${ }^{4}$ Materials Research Institute, Pennsylvania State University, University Park, PA 16802, USA
Häuselmann, pers. comm.), only $450 \mathrm{~m}$ near the cave entrance (Fig. 1b) is of great interest for the occurrence of unusual authigenic cave minerals. To date, over 25 mineral species attributed to six mineral classes (carbonates, oxides and hydroxides, phosphates, sulfates, silicates, and halides) have been documented, some rather unusual for a cave environment (Marincea et al., 2002; Onac et al., 2002, 2005a, 2005b, 2007; Onac \& Effenberger, 2007; Onac \& Tămaş, 2010). Phosphates are by far the best represented class of minerals in Cioclovina Cave. Although most cave phosphates are of immediate interest only to mineralogists, some of these minerals have been studied to achieve an improved understanding of $\mathrm{PO}_{4}{ }^{3-}$ and trace element sources, hydrogeological, and speleogenetic processes.

The aim of this study is to provide further insight into the origin, crystallization sequence, and source of the elements that form(ed) collinsite, atacamite, and kröhnkite by integrating single-crystal $\mathrm{X}$-ray diffraction, electron microprobe analyses (EMPA), scanning electron microscope (SEM) imaging, and stable isotope measurements. In addition, the paper reconsiders two misidentified minerals during the preliminary screening undertaken by Onac et al. (2002): atacamite as paratacamite and kröhnkite as sampleite. 


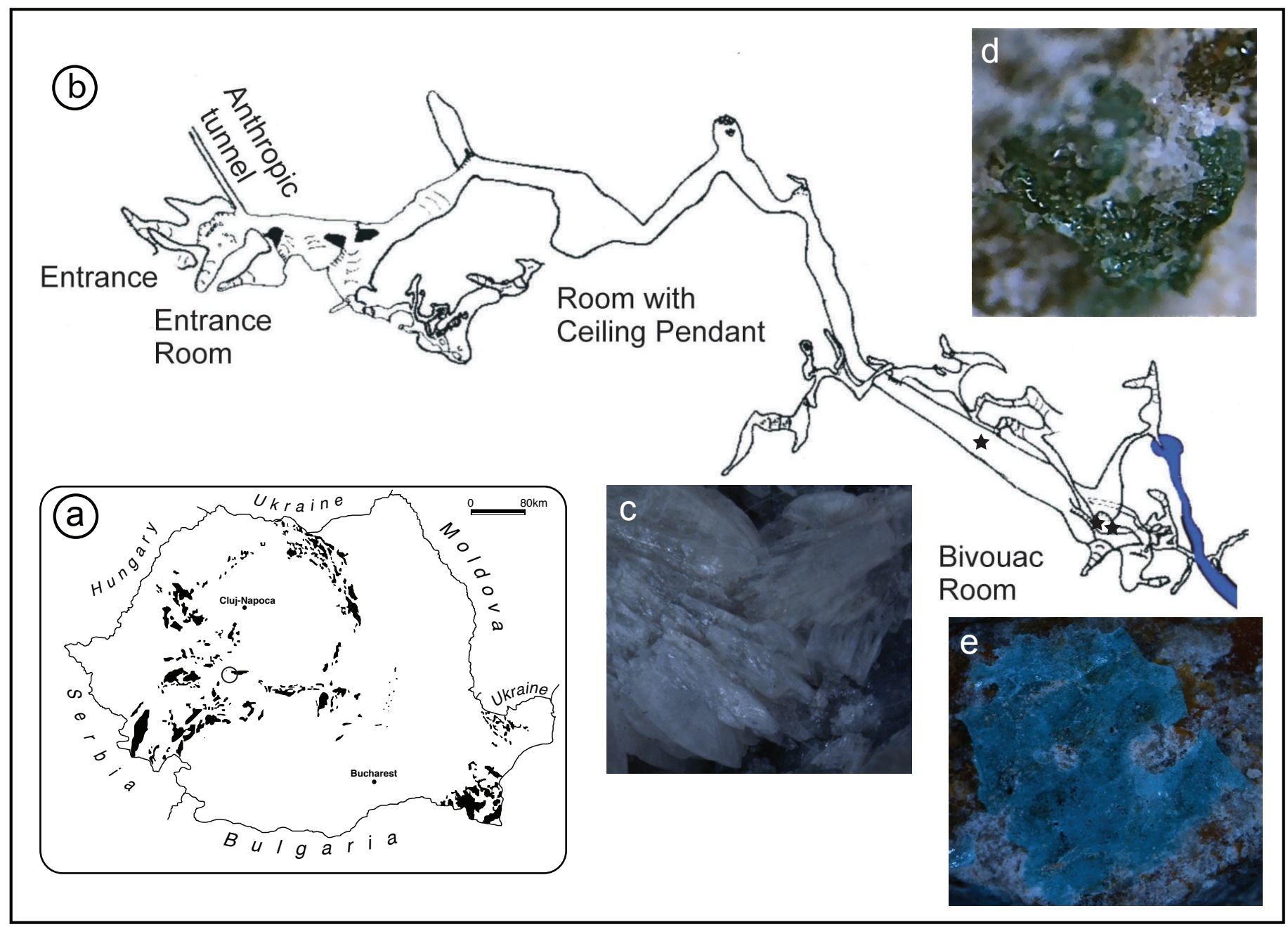

Fig. 1. Location map of Cioclovina Cave (a); map of the cave and sampling sites (b); collinsite (c); atacamite (d); kröhnkite (e).

\section{STUDY AREA SETTING}

The investigated site is on the boundary between the Sebeş-Lotru Series to the east (plagiogneisses and micaschists of Late Precambrian age) and the Alpine sedimentary sequence (Permian and Early Jurassic to Late Cretaceous) to the west (Stilla, 1981). Concentrations of sphalerite, galenite, and pyrite with various amounts of chalcopyrite, bournonite, tetrahedrite, magnetite, hematite, greigite, etc., occurs in vein- and lens-like bodies within the metamorphites of Sebes-Lotru Series (Udubaşa \& Hârtopanu, 1992). Cioclovina Uscată (Dry Cioclovina) Cave is formed in the Early Cretaceous reef limestones of the Luncani karst platform (Mitrofan, 1979) and is part of the larger Ponorici-Cioclovina cu Apă cave system (Tomuş, 1999).

Although several previous studies of the PonoriciCioclovina region have addressed hydrogeological issues, dye tracing experiments, and diving activities (e.g., Mitrofan, 1979; Breban et al., 2003; Bandrabur $\&$ Bandrabur, 2010), only one recent study focused on reconstructing the subaerial paleo-drainage routes based on the sediments accumulated in Cioclovina Cave (Häuselmann et al., 2010). These authors showed that during the cave evolution, large amounts of nearby allogenic detrital sediments derived from weathering of gneisses, mica-schists, and sandstones were deposited along the main cave passage by streams flowing from the east and south of the cave.
At times when stable climate conditions prevailed at the surface, river run-off was low, and therefore, thick guano layers accumulated along the cave passages on top of the previously laid down siliciclastic deposits. Subsequently, washed-in sediments buried each of these organic layers. Due to the presence of interbedded guano material, the sandy-silty sediments were highly phosphatized $\left(\sim 35 \% \mathrm{P}_{2} \mathrm{O}_{5}\right)$ generating, within them and at the contact with the limestone bedrock, a suite of interesting minerals (Dumitraş, 2008; Onac \& Tămaş, 2010).

\section{SAMPLES AND METHODS}

Collinsite occurs near the entrance in the Bivouac Room and is closely associated with hydroxylapatiterich crusts in the upper part of the sedimentary sequence (Fig. 1c). Radial blades or lath-like crystals of collinsite line millimeter-sized dissolution cavities developed within the hydroxylapatite crusts (Fig. 2). Atacamite and kröhnkite were collected just outside the Bivouac Room at its southern edge (Fig. 1b). Both minerals were identified within a 0.5 to $1 \mathrm{~m}$ thick layer of white-yellowish soft powdered ardealite, intimately mixed with brushite and gypsum. Atacamite appears as fine-grained and crumbly greenish nodules (millimeter size in diameter) disseminated in the ardealite-rich, upper part of the phosphate sequence (Fig. 1d). Kröhnkite forms sky-blue platy crusts (sub- 
millimeter thick, up to $10 \mathrm{~mm}$ across) on fragments of unweathered limestone in the vicinity of atacamite and around or over minute gypsum aggregates (Fig. 1e). It was found at a depth of 25 to $35 \mathrm{~cm}$ below the present sediment surface.

For definite identification of the rare mineral samples, crystal-structure refinements of atacamite, collinsite, and kröhnkite from the Cioclovina Cave were performed at the University of Vienna (Nonius Kappa-CCD four-circle diffractometer equipped with a $300 \mu \mathrm{m}$ diameter capillary-optics collimator, graphite-monochromatized MoKa radiation). Data taken from small crystal chips were corrected for background, Lorentz and polarization effects, and absorption (multi-scan method). Programs used include COLLECT (Nonius, 1999), DENZO-SMN (Otwinowski \& Minor, 1997), and SHELXL-97 (Sheldrick, 1997, 2008). Experimental details relative to data collection are reported in Tables 1-3.

Fragments of the crust were embedded in epoxy resin and polished for back-scattered electron imaging. The chemical analyses were performed in energyand wavelength-dispersive mode using a JEOL 8900R Superprobe electron microprobe (EMPA) instrument at the Department of Earth Sciences, Florida International University. Analytical conditions were: 15 $\mathrm{kV}, 5$ and $10 \mathrm{nA}$, electron beam diameter 3-5 $\mu \mathrm{m}$ for atacamite and defocussed to $30 \mu \mathrm{m}$ for collinsite and kröhnkite (samples were moved so as to minimize decomposition of the mineral under the beam), WDX: peak count-time 20-60 s, background count-time 15 s. Apatite $\left(\mathrm{CaO}, \mathrm{P}_{2} \mathrm{O}_{5}\right)$, PlagAn65 $\left(\mathrm{Na}_{2} \mathrm{O}, \mathrm{Al}_{2} \mathrm{O}_{3}\right)$, diopside2 (MgO), Cu-15 (CuO), STD357 kaersutite2 $\left(\mathrm{K}_{2} \mathrm{O}, \mathrm{FeO}\right)$, anhydrite $\left(\mathrm{SO}_{3}\right)$, and tugtupite $(\mathrm{Cl})$ served as standards. Water content in collinsite and kröhnkite has been recalculated for charge balance of the idealized chemical formula. The SEM investigations were carried out on a JEOL $6490 \mathrm{LV}$ in the Lisa Muma Weitz Imaging Core Laboratories (University of South Florida). Sulfur and chlorine isotope analysis of all samples was performed at the University of South Florida and University of Arizona, following the methods described in Wynn et al. (2010) and Eastoe et al. (1989, 2007), respectively.

\section{Chemistry}

The chemical composition of the investigated minerals is based on EMPA measurements - seven point analyses for collinsite and kröhnkite and four spot analyses for atacamite. The chemical composition of all investigated samples is close to the ideal formulas: $\mathrm{Cu}_{2}{ }^{2+} \mathrm{Cl}(\mathrm{OH})_{3}, \quad \mathrm{Ca}_{2} \mathrm{Mg}\left(\mathrm{PO}_{4}\right)_{2} \cdot 2 \mathrm{H}_{2} \mathrm{O}$, and $\mathrm{Na}_{2} \mathrm{Cu}\left(\mathrm{SO}_{4}\right)_{2} \cdot 2 \mathrm{H}_{2} \mathrm{O}$, respectively. Only a moderate substitution of $\mathrm{Mg}$ by $\mathrm{Fe}$ (and minor $\mathrm{Al}$ as well) in collinsite is evident. The average composition (in wt\%) obtained for atacamite is $\mathrm{SiO}_{2}=0.02, \mathrm{CaO}=0.03$, $\mathrm{Na}_{2} \mathrm{O}=0.05, \mathrm{P}_{2} \mathrm{O}_{5}=0.01, \mathrm{CuO}=74.40, \mathrm{~K}_{2} \mathrm{O}=0.02$, $\mathrm{SO}_{3}=0.02, \mathrm{Al}_{2} \mathrm{O}_{3}=0.04, \mathrm{FeO}=0.02$, and $\mathrm{Cl}=16.61$ (total $=91.19)$. It is evident that within the limits of analytical error, the investigated material is ideal, end-member atacamite; notably the $\mathrm{Zn}$ content was below the detection limit. The composition of collinsite is: $\mathrm{CaO}=33.61, \mathrm{P}_{2} \mathrm{O}_{5}=41.83, \mathrm{MgO}=10.50, \mathrm{Al}_{2} \mathrm{O}_{3}=$

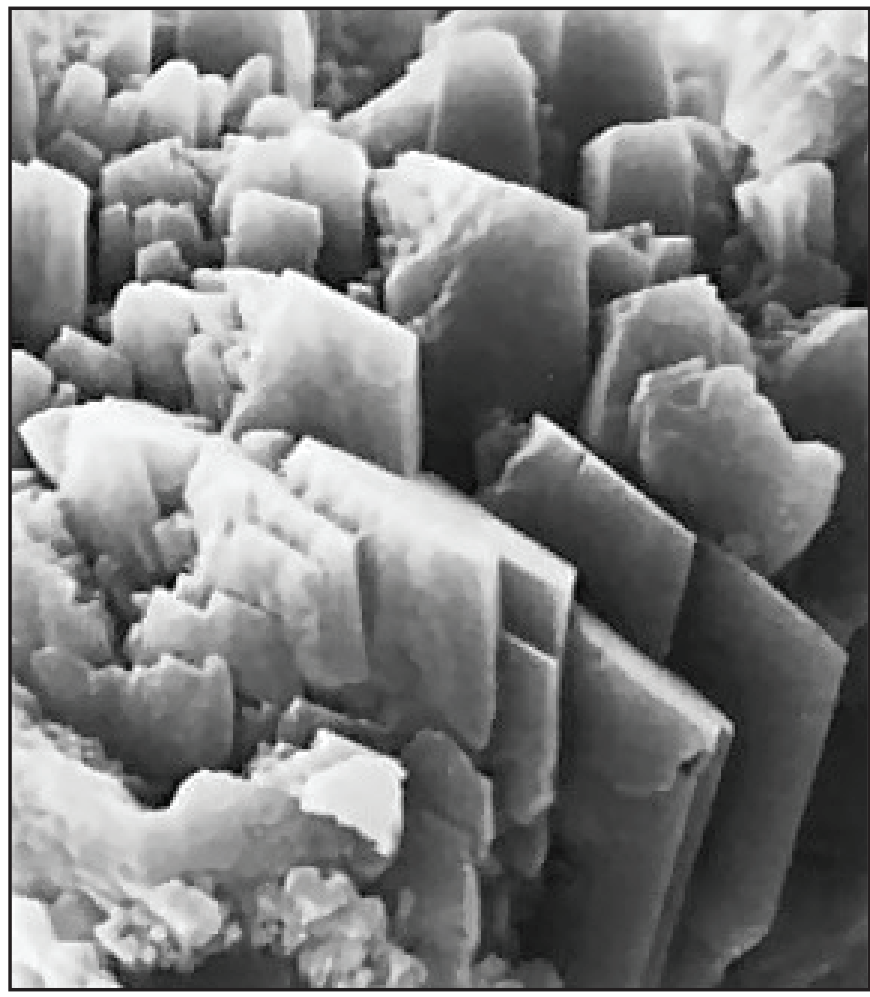

Fig. 2. SEM photograph of lath-like collinsite crystals in vugs of hydroxylapatite crust.

$0.25, \mathrm{FeO}=2.52 .50$, and $\mathrm{H}_{2} \mathrm{O}=10.65$ (total = 99.34); the atomic ratio $\mathrm{Mg}: \mathrm{Fe}^{2+}=0.88: 0.12$. The composition of the kröhnkite is: $\mathrm{SiO}_{2}=0.10, \mathrm{CaO}=0.20, \mathrm{Na}_{2} \mathrm{O}=$ 18.00, $\mathrm{P}_{2} \mathrm{O}_{5}=0.3, \mathrm{MgO}=0.10, \mathrm{CuO}=23.19, \mathrm{~K}_{2} \mathrm{O}=$ $0.30, \mathrm{SO}_{3}=46.97, \mathrm{Al}_{2} \mathrm{O}_{3}=0.10, \mathrm{FeO}=0.20$, and $\mathrm{H}_{2} \mathrm{O}$ $=10.69($ total $=100.15)$.

\section{$X$-ray experiments and refinement of the crystal structure}

For certain identifications, the crystal structures of collinsite, atacamite, and kröhnkite were refined starting from the atomic coordinates given by former authors (Brotherton et al., 1974; Hawthorne \& Ferguson, 1975; Parise \& Hyde, 1986; Yakubovich et al., 2003; Kolitsch \& Fleck, 2005, 2006; Zheng et al., 2005; Herwig \& Hawthorne, 2006; Malcherek \& Schlüter, 2009). The crystal structures compare well with those published by former authors. However, during the present structure refinements all hydrogen bonds were located experimentally. The refinement of collinsite was improved considering a partial substitution of the $\mathrm{Mg}$ atoms by $\mathrm{Fe}$ atoms. Allowing the ratio of the scattering functions for $\mathrm{Mg}$ and $\mathrm{Fe}$ atoms to vary resulted in an occupation $\mathrm{Mg}_{0.88} \mathrm{Fe}_{0.12}$ and gave a better match between observed and calculated intensities; the substitution was proved by the chemical analysis. In kröhnkite, the sodium position shows somewhat large displacement parameters that might be a hint for vacancies; a trial to refine the occupation resulted in $\mathrm{Na}_{0.986(4)}$ which is at the limit of significance. It is to be mentioned that the electron-microprobe analysis showed a number of small impurities that might substitute the $\mathrm{Na}, \mathrm{Cu}$ and $\mathrm{S}$ sites. Details about data collection, refinements and results are compiled in Table 1. The final structural parameters of the three minerals from the Cioclovina Cave are given in Table 
Table 1. Single-crystal X-ray data-collection and structure refinements.

\begin{tabular}{|c|c|c|c|}
\hline & Collinsite & Atacamite & Kröhnkite \\
\hline Chemical formula & $\mathrm{Ca}_{2}(\mathrm{Mg}, \mathrm{Fe})\left(\mathrm{PO}_{4}\right)_{2} \cdot 2 \mathrm{H}_{2} \mathrm{O}$ & $\mathrm{Cu}_{2} \mathrm{Cl}(\mathrm{OH})_{3}$ & $\mathrm{Na}_{2} \mathrm{Cu}\left(\mathrm{SO}_{4}\right)_{2} \cdot 2 \mathrm{H}_{2} \mathrm{O}$ \\
\hline$a[\AA]$ & $5.738(1)$ & $6.035(1)$ & $5.518(1)$ \\
\hline$b[\AA]$ & $6.790(1)$ & $6.872(1)$ & $12.666(2)$ \\
\hline$c[\AA ̊]$ & $5.455(1)$ & $9.126(1)$ & $5.808(1)$ \\
\hline $\mathrm{a}\left[{ }^{\circ}\right]$ & $97.30(1)$ & - & - \\
\hline$\beta\left[{ }^{\circ}\right]$ & $108.56(1)$ & - & $108.45(1)$ \\
\hline $\mathrm{V}\left[{ }^{\circ}\right]$ & $107.27(1)$ & - & - \\
\hline $\mathrm{V}\left[\AA^{3}\right]$ & 186.5 & 378.5 & 385.1 \\
\hline space group & $P \overline{1}$ & Pnma & $2{ }_{1} / c$ \\
\hline $\mathrm{Z}$ & 1 & 4 & 2 \\
\hline$\rho_{\text {calc }}\left[\mathrm{g} \mathrm{cm}^{-3}\right] / \mu(\operatorname{MoKa})\left[\mathrm{mm}^{-1}\right]$ & $2.98 / 2.3$ & $3.75 / 11.8$ & $2.91 / 3.5$ \\
\hline crystal dimensions $(\mu \mathrm{m})$ & $50 \times 80 \times 100$ & $50 \times 60 \times 160$ & $35 \times 50 \times 180$ \\
\hline $\begin{array}{l}\text { range of data collection } \\
( \pm h \pm k \pm l)\left[{ }^{\circ}\right]\end{array}$ & $3<2 \vartheta<70$ & $3<2 \vartheta<70$ & $3<2 \vartheta<70$ \\
\hline $\begin{array}{l}\text { number of images / rotation } \\
\text { angle per image }\left[{ }^{\circ}\right]\end{array}$ & $759 / 2$ & $505 / 2$ & $575 / 2$ \\
\hline scan mode: $\varphi$-scans & at 12 distinct $\omega$-angles & $\begin{array}{lr}\text { at } 9 & \text { distinct } \\
\omega \text {-angles } & \end{array}$ & $\begin{array}{l}\text { at } 10 \text { distinct } \\
\omega \text {-angles }\end{array}$ \\
\hline $\begin{array}{l}\text { scan time }\left[\mathrm{s} /{ }^{\circ}\right] / \text { frame size } \\
\text { (binned mode) }\end{array}$ & 60 / $621 \times 576$ pixels & $70 / 621 \times 576$ pixels & $\begin{array}{l}140 \text { / } 621 x \quad 576 \\
\text { pixels }\end{array}$ \\
\hline $\begin{array}{l}\text { detector-to-sample distance } \\
{[\mathrm{mm}]}\end{array}$ & 30 & 30 & 30 \\
\hline measured reflections & 3341 & 6493 & 3407 \\
\hline $\begin{array}{l}\text { unique reflections }(n) / \text { observed } \\
\text { reflections with } F_{o}>4 \sigma\left(F_{0}\right)\end{array}$ & 1635 / 1532 & $884 / 845$ & 1694 / 1506 \\
\hline$R_{\text {int }}=\Sigma \mid F_{o}^{2}-F_{o}^{2}($ mean $) \mid / \Sigma F_{o}^{2}$ & 0.014 & 0.028 & 0.016 \\
\hline extinction parameter & $0.131(11)$ & $0.050(3)$ & $0.016(3)$ \\
\hline $\begin{array}{l}R 1=\Sigma\left(|| F_{o}|-| F_{c}||\right) / \Sigma F_{o} \\
\text { (unique/observed reflections) }\end{array}$ & $0.023 / 0.022$ & $0.022 / 0.020$ & $0.033 / 0.027$ \\
\hline$w \mathrm{R} 2=\left[\Sigma\left(F_{o}^{2}-F_{c}^{2}\right)^{2} / \Sigma \mid \omega F_{o}^{4}\right]^{1 / 2}$ & 0.060 & 0.057 & 0.077 \\
\hline GooF $=\left\{\Sigma\left[w\left(F_{o}^{2}-F_{c}^{2}\right)^{2}\right] /(n-p)\right\}^{0.5}$ & 1.097 & 1.070 & 1.107 \\
\hline $\begin{array}{l}\max \Delta / \sigma / \text { number of variable } \\
\text { parameters }(p)\end{array}$ & $<0.001 / 80$ & $<0.001 / 42$ & $<0.001 / 80$ \\
\hline $\begin{array}{l}\text { final difference Fourier map } \\
{\left[\mathrm{e}^{-3}\right]}\end{array}$ & -0.60 to +0.46 & -0.89 to +0.85 & -0.96 to +0.50 \\
\hline weighting parameters a / b & $0.032 / 0.080$ & $0.040 / 0.120$ & $0.043 / 0.150$ \\
\hline
\end{tabular}

NONIUS four-circle diffractometer equipped with a CCD detector and a $300 \mu \mathrm{m}$ capillary-optics collimator (Mo tube, graphite monochromator). Unit-cell parameters were obtained by least-squares refinements of $2 \vartheta$ values. Corrections for Lorentz, polarization and absorption effects (multi-scan method); complex scattering functions for neutrally charged atoms (Wilson, 1992); programs Collect (Nonius, 1999, Otwinowski and Minor, 1997), SHELXL-97 (Sheldrick, 1997, 2008). $\left.w=1 /\left\{\sigma^{2}\left(F_{o}^{2}\right)+[\mathrm{a} \times P]^{2}+\mathrm{b} \times P\right\} ; P=\left(\left[\max \left(0, F_{o}^{2}\right)\right]+2 \times F_{c}^{2}\right) / 3\right]$

2 ; interatomic bond distances and the geometry of the hydrogen bonds in Table 3. Further details are available from the Fachinformationszentrum Karlsruhe, D-76344 Eggenstein-Leopoldshafen (crystal@FIZKarlsruhe) on quoting the deposition number CSD 422592 (atacamite), 422593 (kröhnkite), and 422594 (collinsite).
In the past, a number of compounds with the general formula $M 1_{\mathrm{n}}{ }^{1+, 2+} M 2^{2+, 3+}\left(X^{5+, 6+} \mathrm{O}_{4}\right)_{2} \cdot 2 \mathrm{H}_{2} \mathrm{O}$ containing infinite $\mathrm{M} 2\left(\mathrm{XO}_{4}\right)_{2}\left(\mathrm{H}_{2} \mathrm{O}\right)$ chains of alternating corner-sharing $\mathrm{XO}_{4}$ tetrahedra and $\mathrm{MO}_{6}$ polyhedra have been investigated (for extended discussions and comparisons cf. Hawthorne \& Ferguson (1975); Yakubovich et al., 2003; Wildner \& Stoilova (2003); Kolitsch \& Fleck, 
Table 2. Fractional atomic coordinates and displacement parameters. The anisotropic displacement parameters are defined as: exp $\left[-2 \pi^{2} \sum_{i=1}^{3} \sum_{j=1}^{3} U_{i j} a_{i}^{*} a_{j}^{*} h_{i} h_{j}\right]$.

$\begin{array}{llllllllllllll}\text { atom } & \text { site } & x & y & z & U_{\text {equiv }} / U_{\text {iso }} & U_{11} & U_{22} & U_{33} & U_{23} & U_{13} & U_{12} \\ \text { symmetry } & & & & & \end{array}$

\section{collinsite, $\mathrm{Ca}_{2}(\mathrm{Mg}, \mathrm{Fe})\left(\mathrm{PO}_{4}\right)_{2} \cdot 2 \mathrm{H}_{2} \mathrm{O}$}

$\begin{array}{lllllllllll}\mathrm{Ca} & 0.30338(4) & 0.75898(3) & 0.65415(4) & 0.01155(7) & 0.01069(10) & 0.01251(10) & 0.01106(10) & 0.00180(7) & 0.00464(7) & 0.00347(7)\end{array}$

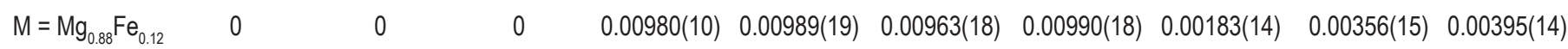

$\begin{array}{lllllllllll}\mathrm{P} & 0.33309(5) & 0.24378(4) & 0.66332(5) & 0.00831(7) & 0.00815(12) & 0.00864(11) & 0.00815(12) & 0.00245(8) & 0.00281(8) & 0.00311(8)\end{array}$

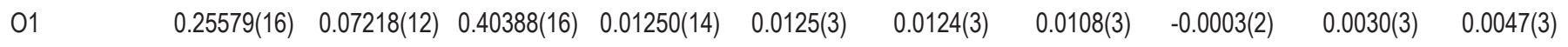

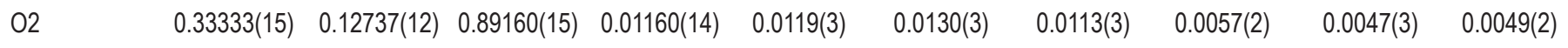

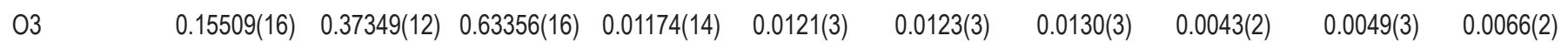

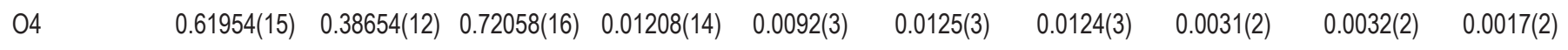

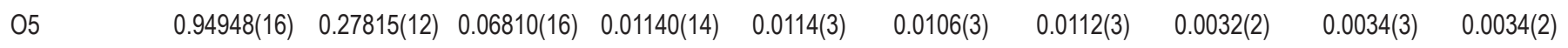

$\mathrm{H} 1 \quad 0.857(5) \quad 0.306(4) \quad-0.067(5) \quad 0.038(6)$

$\mathrm{H} 2 \quad 0.086(6) \quad 0.360(4) \quad 0.114(6) \quad 0.059(9)$

\section{atacamite, $\mathrm{Cu}_{2} \mathrm{Cl}(\mathrm{OH})_{3}$}

$\begin{array}{lcccccccccc}\text { Cu1 } & 0 & 0 & 0 & 0.01182(10) & 0.01328(15) & 0.01028(14) & 0.01188(13) & -0.00135(5) & 0.00095(6) & -0.00139(6) \\ \text { Cu2 } & 0.19060(4) & 1 / 4 & 0.25523(2) & 0.01185(9) & 0.01012(14) & 0.01118(13) & 0.01426(13) & 0 & -0.00180(6) & 0 \\ \mathrm{Cl} & 0.35127(7) & 3 / 4 & 0.05560(5) & 0.01659(11) & 0.01426(17) & 0.01795(19) & 0.01757(19) & 0 & -0.00204(13) & 0 \\ \mathrm{O} 1 & 0.1504(3) & 1 / 4 & -0.00205(18) & 0.0139(2) & 0.0128(6) & 0.0110(6) & 0.0178(6) & 0 & 0.0039(4) & 0 \\ \mathrm{O} 2 & 0.44098(14) & 0.06548(13) & 0.28844(10) & 0.01273(17) & 0.0139(4) & 0.0107(4) & 0.0136(4) & -0.0007(3) & -0.0012(3) & 0.0000(3) \\ \mathrm{H} 1 & 0.185(6) & 1 / 4 & -0.042(4) & 0.030(14) & & & & & & \\ \mathrm{H} 2 & 0.419(6) & -0.017(6) & 0.231(3) & 0.039(8) & & & & & & \end{array}$

\section{kröhnkite, $\mathrm{Na}_{2} \mathrm{Cu}\left(\mathrm{SO}_{4}\right)_{2} \cdot 2 \mathrm{H}_{2} \mathrm{O}$}

$\mathrm{Cu}$

$\mathrm{Na}_{0.986(4)}$

$S$

01

02

03

04

05

H1

$\mathrm{H} 2$
$0 \quad 0$

$\begin{array}{cccccccccc}0.57086(14) & 0.12468(6) & 0.20982(15) & 0.0240(2) & 0.0206(3) & 0.0228(4) & 0.0321(4) & -0.0021(3) & 0.0134(3) & -0.0008(2) \\ 0.23759(6) & 0.11804(3) & 0.54450(6) & 0.01317(9) & 0.01169(13) & 0.01253(16) & 0.01454(16) & -0.00031(11) & 0.00309(11) & 0.00029(10) \\ 0.00475(19) & 0.17262(10) & 0.4885(2) & 0.0199(2) & 0.0150(4) & 0.0190(6) & 0.0246(6) & 0.0005(4) & 0.0046(4) & 0.0055(3) \\ 0.2910(2) & 0.05750(9) & 0.7843(2) & 0.0184(2) & 0.0168(4) & 0.0185(5) & 0.0196(5) & 0.0047(4) & 0.0053(4) & 0.0008(4) \\ 0.2324(2) & 0.04296(10) & 0.3317(2) & 0.0197(2) & 0.0144(4) & 0.0210(5) & 0.0215(5) & -0.0071(4) & 0.0025(4) & 0.0019(4) \\ 0.4381(2) & 0.19254(10) & 0.5610(2) & 0.0194(2) & 0.0167(4) & 0.0177(5) & 0.0233(5) & -0.0003(4) & 0.0053(4) & -0.0042(4) \\ 0.8316(2) & 0.13419(9) & 0.9513(2) & 0.0165(2) & 0.0149(4) & 0.0138(5) & 0.0184(5) & -0.0007(4) & 0.0017(4) & -0.0009(4) \\ 0.910(5) & 0.187(2) & 0.972(5) & 0.026(6) & & & & & & \\ 0.743(5) & 0.139(2) & 0.825(5) & 0.029(7) & & & & & & \end{array}$


Table 3. Interatomic bond lengths $(\AA)$ and bond angles $\left({ }^{\circ}\right)$.

\section{collinsite, $\mathrm{Ca}_{2}(\mathrm{Mg}, \mathrm{Fe})\left(\mathrm{PO}_{4}\right)_{2} \cdot \mathbf{2} \mathrm{H}_{2} \mathrm{O}$}

$\begin{array}{llllll}\mathrm{Ca}-\mathrm{O} 4 & 2.3817(9) & (\mathrm{Mg}, \mathrm{Fe})-\mathrm{O} 5 & 2.0011(8), 2 \mathrm{x} & \mathrm{P}-\mathrm{O} 3 & 1.5199(9) \\ \mathrm{Ca}-\mathrm{O} 5 & 2.3965(9) & (\mathrm{Mg}, \mathrm{Fe})-\mathrm{O} 1 & 2.1185(9), 2 \mathrm{x} & \mathrm{P}-\mathrm{O} 4 & 1.5456(9) \\ \mathrm{Ca}-\mathrm{O} 3 & 2.4085(10) & (\mathrm{Mg}, \mathrm{Fe})-\mathrm{O} 2 & 2.1503(8), 2 \mathrm{x} & \mathrm{P}-\mathrm{O} 2 & 1.5573(8) \\ \mathrm{Ca}-\mathrm{O} 3 & 2.4759(9) & & & \mathrm{P}-\mathrm{O} 1 & 1.5594(9) \\ \mathrm{Ca}-\mathrm{O} 2 & 2.5203(9) & & & \\ \mathrm{Ca}-\mathrm{O} 1 & 2.5871(10) & & & & \\ \mathrm{Ca}-\mathrm{O} 2 & 2.5969(9) & & & & \\ \mathrm{Ca}-\mathrm{O} 1 & 2.7030(9) & & & & \end{array}$

Hydrogen bonds

$\begin{array}{lcccccccc}\text { Donor } & \mathrm{H} \text { atom } & \text { Acceptor } & \mathrm{D}-\mathrm{H} & \mathrm{H} \cdots \mathrm{A} & \mathrm{D} \cdots \mathrm{A} & \mathrm{D}-\mathrm{H} \cdots \mathrm{A} & \text { water molecule } \\ \text { O5 } & \mathrm{H} 1 & \mathrm{O} 4 & 0.84(3) & 1.76(3) & 2.5723(12) & 162(3) & \mathrm{H} 1-\mathrm{O} 5-\mathrm{H} 2 & 106(2) \\ \mathrm{O} 5 & \mathrm{H} 2 & \mathrm{O} 4 & 0.75(3) & 1.88(3) & 2.6083(12) & 162(3) & \mathrm{O} 4-\mathrm{O} 5-\mathrm{O} 4 & 105.78(4)\end{array}$

\section{atacamite, $\mathrm{Cu}_{2} \mathrm{Cl}(\mathrm{Ox})_{3}$}

$\begin{array}{llllll}\mathrm{Cu} 1-\mathrm{O} 1 & 1.9432(8), 2 \mathrm{x} & \mathrm{Cu} 2-\mathrm{O} 2 & 1.9957(9), 2 \mathrm{x} & \mathrm{Cl}-\mathrm{Cu} 2 & 2.7528(6) \\ \mathrm{Cu} 1-\mathrm{O} 2 & 2.0142(10), 2 \mathrm{x} & \mathrm{Cu} 2-\mathrm{O} 2 & 2.0091(9), 2 \mathrm{x} & \mathrm{Cl}-\mathrm{Cu} 1 & 2.7754(5), 2 \mathrm{x} \\ \mathrm{Cu} 1-\mathrm{C} 1 & 2.7754(5), 2 \mathrm{x} & \mathrm{Cu} 2-\mathrm{O} 1 & 2.3605(17) & \mathrm{Cl}-\mathrm{H} 2 & 2.30(3), 2 \mathrm{x} \\ \mathrm{Cu} 2-\mathrm{Cl} & 2.7528(6) & \mathrm{Cl}-\mathrm{H} 1 & 2.80(4) & & \\ & & \mathrm{Cl} \cdots \mathrm{O} & 3.047(2) & & \end{array}$

Hydrogen bonds

$\begin{array}{lcccccc}\text { Donor } & \mathrm{H} \text { atom } & \text { Acceptor } & \mathrm{D}-\mathrm{H} & \mathrm{H} \cdots \mathrm{A} & \mathrm{D} \cdots \mathrm{A} & \mathrm{D}-\mathrm{H} \cdots \mathrm{A} \\ \mathrm{O} 1 & \mathrm{H} 1 & \mathrm{Cl} & 0.42(4) & 2.80(4) & 3.0466(18) & 122(6) \\ \mathrm{O} 2 & \mathrm{H} 2 & \mathrm{Cl} & 0.78(3) & 2.30(3) & 3.0836(10) & 178(4)\end{array}$

\section{kröhnkite, $\mathrm{Na}_{2} \mathrm{Cu}\left(\mathrm{SO}_{4}\right)_{2} \cdot 2 \mathrm{H}_{2} \mathrm{O}$}

$\begin{array}{llllll}\mathrm{Na}-\mathrm{O} 5 & 2.3884(14) & \mathrm{Cu}-\mathrm{O} 5 & 1.9147(12), 2 \mathrm{x} & \mathrm{S}-\mathrm{O} 1 & 1.4036(11) \\ \mathrm{Na}-\mathrm{O} 2 & 2.4269(14) & \mathrm{Cu}-\mathrm{O} 3 & 2.0218(12), 2 \mathrm{x} & \mathrm{S}-\mathrm{O} 4 & 1.4340(12) \\ \mathrm{Na}-\mathrm{O} 3 & 2.4285(15) & \mathrm{Cu}-\mathrm{O} 2 & 2.4395(21), 2 \mathrm{x} & \mathrm{S}-\mathrm{O} 2 & 1.5347(12) \\ \mathrm{Na}-\mathrm{O} 4 & 2.4986(15) & & \mathrm{S}-\mathrm{O} 3 & 1.5525(12) \\ \mathrm{Na}-\mathrm{O} 1 & 2.5037(14) & & & \\ \mathrm{Na}-\mathrm{O} 4 & 2.5262(15) & & & \\ \mathrm{Na}-\mathrm{O} 2 & 2.6078(15) & & & \end{array}$

Hydrogen bonds

Donor $\mathrm{H}$ atom Acceptor $\mathrm{D}-\mathrm{H} \quad \mathrm{H} \cdots \mathrm{A} \quad \mathrm{D} \cdots \mathrm{A} \quad \mathrm{D}-\mathrm{H} \cdots \mathrm{A} \quad$ water molecule

$\begin{array}{lllllllll}\mathrm{O} 5 & \mathrm{H} 1 & \mathrm{O} 1 & 0.78(3) & 1.85(3) & 2.611(2) & 164(3) & \mathrm{H} 1-\mathrm{O} 5-\mathrm{H} 2 & 104(3) \\ \mathrm{O} 5 & \mathrm{H} 2 & \mathrm{O} 4 & 0.74(3) & 2.00(3) & 2.699(2) & 156(3) & \mathrm{O} 1-\mathrm{O} 5-\mathrm{O} 4 & 89.37(5)\end{array}$


2005, 2006; Zheng et al., 2005; Herwig \& Hawthorne, 2006 and references therein). In these papers the $M 2\left(\mathrm{XO}_{4}\right)_{2}\left(\mathrm{H}_{2} \mathrm{O}\right)$ chains and their connections are discussed extensively. Alternatively, the crystal structure of collinsite, $\mathrm{Ca}_{2} \mathrm{Mg}\left(\mathrm{PO}_{4}\right)_{2} \cdot 2 \mathrm{H}_{2} \mathrm{O}^{-}$, can be described as consisting of layers parallel to (110) formed by edge sharing $\mathrm{CaO}_{8}$ polyhedra; six-member rings host the hydrogen bonds. They are linked by $\mathrm{MgO}_{6}$ octahedra and phosphate tetrahedra (Fig. 3).

Kröhnkite, $\mathrm{Na}_{2} \mathrm{Cu}\left(\mathrm{SO}_{4}\right)_{2} \cdot 2 \mathrm{H}_{2} \mathrm{O}$, is formed by ribbons of pentagonal pyramids of $\mathrm{NaO}_{7}$ and elongated tetragonal pyramids of $\mathrm{CuO}_{6}$ running parallel to [100]. The $\mathrm{NaO}_{7}$ polyhedra share edges among each other and with the $\mathrm{CuO}_{6}$ polyhedra. The ribbons are linked by the hydrogen bonds of the water molecule and the sulfate tetrahedra (Fig. 4).

Atacamite, $\mathrm{Cu}_{2}{ }^{2+} \mathrm{Cl}(\mathrm{OH})_{3}$, is characterised by the [4+2] distorted $\mathrm{Cu} 1(\mathrm{OH})_{4} \mathrm{Cl}_{2}$ and $\mathrm{Cu} 2(\mathrm{OH})_{5} \mathrm{Cl}$ polyhedra (Jahn-Teller effect). Chains of corner-sharing $\mathrm{Cu} 1 \mathrm{O}_{4}$ squares running parallel to [010] and chains of edge sharing $\mathrm{Cu}_{2} \mathrm{O}_{4}$ squares running parallel to [100] form a three-dimensional network with the $\mathrm{Cl}$ atoms and hydrogen bonds located in the channels running parallel to the $\mathrm{Cu} 1(\mathrm{OH})_{4} \mathrm{Cl}_{2}$ chains.

\section{DISCUSSION}

Although powder X-ray diffraction is an excellent screening technique for the detection of minerals, the use of this method can misidentify minerals due to interferences and interpretive errors, especially when limited amount of material is available and admixtures cannot be sorted out. Such occurred during the preliminary mineralogical screening undertaken by Onac et al. (2002). In the present study, we have shown that two minerals, initially identified as paratacamite and sampleite are instead atacamite and kröhnkite. Both these minerals are copper-bearing species; their presence in a cave environment is unusual. This is, however, not the first time that $\mathrm{Cu}$ minerals have been reported in caves. Along with the more common minerals malachite, azurite, chalcanthite, and aurichalcite, a number of very uncommon species, including sampleite, aubertite, brochantite, cyanotrichite, devilline, and rosasite were documented from a variety of cave settings worldwide (Hill \& Forti, 1997 and references therein). In most cases the formation of these phases has been explained as ore body-derived. Atacamite was earlier reported from Jingemia Cave, Western Australia (Bridge et al., 1978), Mbobo Mkulu Cave, South Africa (Martini, 1980), and recently from Cueva de la Mina Chulacao (Atacama, Chile) by De Waele et al. (2009). The presence of atacamite at the last occurrence is expected given the low relative humidity ( $\mathrm{RH}$ ) values (below 25\%) outside and inside the cave. However, the other atacamite samples (including the one discussed in this paper) are reported from much colder caves with RH well above 50\%. In Cioclovina Cave, the temperature in the Bivouac Room is a constant $\sim 9^{\circ} \mathrm{C}$ and the $\mathrm{RH}$ varies between 75 and $95 \%$ (Onac et al., 2002).

For all cave occurrences of atacamite, $\mathrm{Cu}^{2+}$ was either mobilized in low-pH conditions from near-by sources of copper-rich minerals or it was leached from the cave host rock or volcanic ash layers overlying halite deposits (e.g., Cueva de la Mina Chulacao;

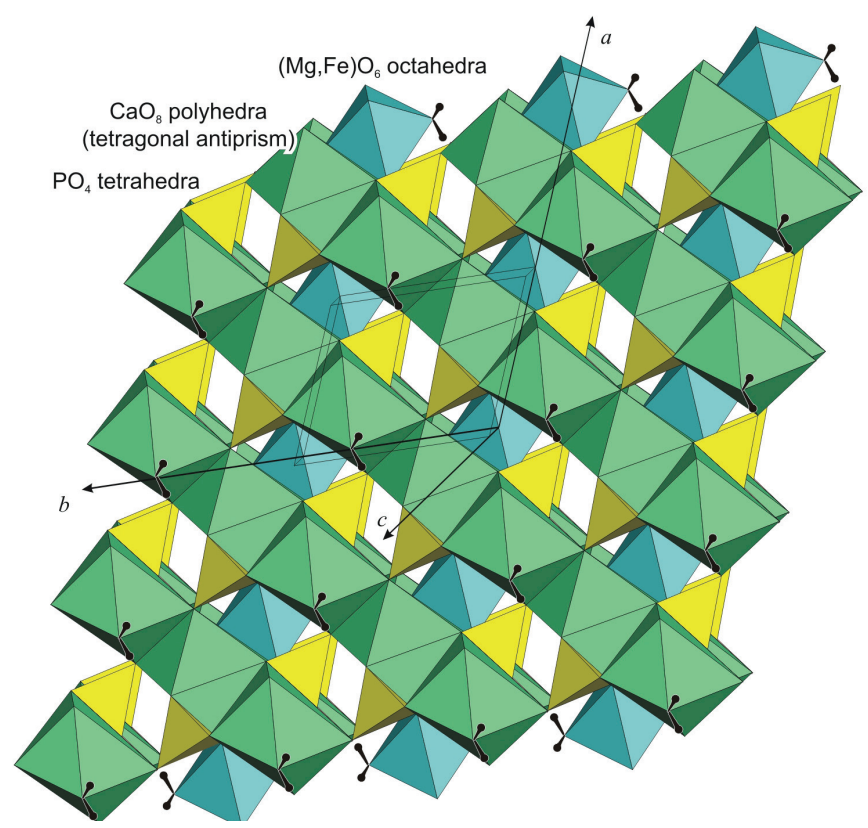

Fig. 3. Crystal structure of collinsite.

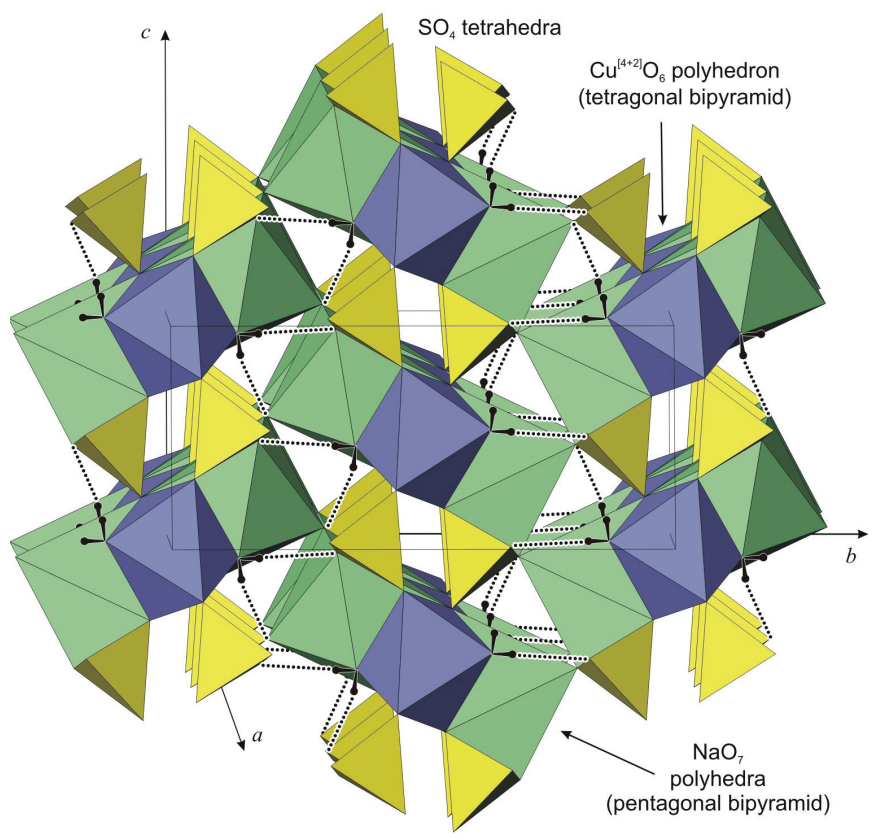

Fig. 4. Projection along [100] of the crystal structure of kröhnkite.

De Waele et al., 2009). Some minor ore bodies identified north-east of the Cioclovina Cave (Udubaşa \& Hârtopanu, 1992) might be the source of $\mathrm{Cu}^{2+}$ for the atacamite in this cave. Based on a thorough investigation of the clastic sediments accumulated in Cioclovina Cave, Häuselmann et al. (2010) suggested that these sediments were transported into the cave by a paleo-surface stream flowing from NE to SW. Guano and/or meteoric water seem to supply chlorine at the Australian and South African cave sites. In the salt karst of Cordillera de la Sal (Atacama, Chile), $\mathrm{Cl}^{-}$is leached from the halite deposits hosting the cave.

The $\mathrm{Cl}$ source in the Cioclovina atacamite has been investigated using stable isotopes technique. In an attempt to identify the chlorine origin, three samples of atacamite and two Lower Cretaceous limestone 
fragments were analyzed to determine their ${ }^{37} \mathrm{Cl} /{ }^{35} \mathrm{Cl}$ ratios. The $\delta^{37} \mathrm{Cl}$ values for atacamite $( \pm 0.1 \%$ precision) range from -0.2 to $+0.1 \%$ relative to the standard mean ocean chloride (SMOC), whereas, both measured Cretaceous sediments gave identical values of $-0.1 \%$ o (SMOC). The $\delta^{37} \mathrm{Cl}$ values obtained for atacamite are indicative of nonmagmatic origin for the chloride (Kaufmann et al., 1984; Sharp et al., 2007). The similar values of $\delta^{37} \mathrm{Cl}$ in atacamite and Cretaceous limestone suggest that the exposed Lower Cretaceous reef complex was leached by meteoric water generating elevated concentrations of chloride in the percolating groundwater and thus its isotopic signature is preserved in the atacamite samples.

Some micrograms of sky-blue kröhnkite (collected from a $0.8 \times 0.9 \mathrm{~mm}$ crust) were used to obtain chlorine isotope data following the analytical procedures outlined in Eastoe et al. (1989). All three kröhnkite samples have $\delta^{37} \mathrm{Cl}$ values near $0.0 \%$, similar to atacamite, suggesting Lower-Cretaceous limestonederived chlorine. This was further conformed by the range of $\delta^{34} \mathrm{~S}$ (16.3 to $17.8 \%$ oDT), which overlap the predicted $\delta^{34} \mathrm{~S}$ values of Cretaceous marine evaporites. The most probable source for $\mathrm{Na}^{+}$are the Precambrian and Permian weathered rocks (gneiss, micaschist, and sandstone) outcropping near the cave, on both sides of the former surface paleo-drainage direction.

Acid conditions are created as oxygenated ground water percolates through the cave sediments and oxidizes sulfide minerals. When these acid solutions came in contact with limestone or cave sediment, a $\mathrm{pH}$ increase was triggered, resulting in the over-saturation and subsequent precipitation of ardealite, brushite, and gypsum. Under oxidizing low-pH conditions, the $\mathrm{Cu}^{2+}$ cation is stable in solution. When the $\mathrm{pH}$ is in the range of 4.8 to 5.6 , due to neutralization reaction in a Ca-depleted environment (depending on the $\mathrm{Cl}^{-}$and $\mathrm{Cu}^{2+}$ activities), atacamite and kröhnkite will precipitate. Once formed, atacamite is stable between pH 6.75 and 7.55 (Eggenkamp \& Schuiling, 1995). Therefore, the neutralization potential of the host-rock controls the sequence of minerals that will form.

Previously, collinsite had only been reported from two caves, Blue Lagoon Cave in western Transvaal (South Africa) by Martini \& Moen (1998) and from Altar Cave on San Salvador Island (Bahamas) by Onac et al. (2001). A preliminary note on the presence of collinsite in Cioclovina Cave was published by Onac et al. (2002). At that time, however, the mineral identification relied only on X-ray powder diffraction without any further insights into its physical or chemical characteristics. The single-crystal X-ray and EMPA examinations reported here confirmed the presence of this rare phosphate in Cioclovina Cave. At all three locations where collinsite has been positively identified, it presumably originated from the reaction of phosphatic solutions leached from bat guano with limestone or cave sediments. The collinsite in Cioclovina Cave is underlain by residual acid calcium phosphates (e.g., brushite and ardealite), which in turn are underlain by monetite. Based on the mineral association, we assume that it formed along with hydroxylapatite and leucophosphite from basic to near-neutral

\section{CONCLUSIONS}

The Cioclovina Cave is notable for the coexistence of low-temperature (a variety of carbonates, sulfates, and phosphates) and high-temperature (berlinite and hydroxylellestadite) minerals. Single-crystal Xray investigations, EMPA, and structure refinement confirmed the presence of collinsite, atacamite, and kröhnkite, in the mineral assemblage of the phosphate-rich deposit of Cioclovina Cave.

The $\delta^{34} \mathrm{~S}$ (CTD) and $\delta^{37} \mathrm{Cl}$ (SMOC) values for atacamite and kröhnkite suggest a Lower-Cretaceous limestone-derived chlorine and sulfate source. These minerals are indicators of an acidic environment, caused by the oxidation of various sulfides disseminated within the cave sediments. This is the first report of kröhnkite from a cave environment. Based on textural evidence and geochemical considerations, we deduced the following general sequence of crystallization in the phosphate-rich sediment sequence containing the Cu-bearing minerals: ardealite-brushite-(gypsum)-atacamite/kröhnkite. This suggests that the observed mineral paragenesis is controlled by $\mathrm{pH}$ (i.e., neutralization potential of the host-rock or cave sediment mineralogy) and the availability of $\mathrm{Ca}, \mathrm{Cl}$, $\mathrm{Cu}$, and $\mathrm{Na}$.

Despite many interesting and controversial findings reported from the Cioclovina Uscata Cave over the past 10 years, most of these minerals are endemic. Their occurrence and abundance is limited to particular geochemical microenvironments, clearly illustrating the complicated nature of secondary cave mineral formation in complex cave sediment settings.

\section{ACKNOWLEDGEMENTS}

The Grădiştea Muncelului-Cioclovina Natural Park is thanked for granting permission to collect samples for this study. Thoughtful comments by Dr. A.R. Kampf, an anonymous reviewer, and editor Jo De Waele are gratefully acknowledged. This research project was funded through the Romanian National University Research Council (\#1696; fieldwork and preliminary analysis) and an internal University of South Florida grants to BPO (SEM and EMPA). We thank C.M. Puscaş for collecting collinsite SEM microphotographs.

\section{REFERENCES}

Alexandrescu E., Olariu A., Skog G., Stenström K. \& Hellborg R., 2010 - Os fossiles humains des grottes Muierii et Cioclovina, Roumanie. L'Anthropologie, 114: 341-353.

Bandrabur G. \& Bandrabur R., 2010 - Sebes Mountains. In: Orăşeanu I. \& Iurkiewicz A. (Eds.), Karst hydrogeology of Romania, Belvedere, Oradea, 77-88. Breban R., Şerban M., Viehmann I. \& Băicoană M., 2003 - The history of guano-phosphate exploration and discovery of human fossils in Ciclovina Uscata Cave. Proteus, Hunedoara, 154 pp. (in Romanian).

Bridge P.J., Pryce M.W., Clarke R.M. \& Costello M.B., 1978 - Sampleite from Jingemia Cave, Western Aus- 
tralia. Mineralogical Magazine, 42: 369-371.

Brotherton P.D., Maslen E.N., Pryce M.W. \& White A.H., 1974 - Crystal structure of collinsite. Austral. J. Chem., 27: 653-656.

De Waele J., Forti P., Picotti V., Galli E., Rossi A., Brook G., Zini L. \& Cucchi F., 2009 - Cave deposits in Cordillera de la Sal (Atacama, Chile). GeoActa Spec. Publ., 2: 97-111.

Dumitraş D.-G., 2008 - Mineralogical associations in the sediments from Cioclovina Uscată Cave. Unpubl. PhD Thesis, University of Bucharest (in Romanian). Eastoe C.J., Guilbert J.M. \& Kaufmann R.S., 1989 Preliminary evidence for fractionation of stable chlorine isotopes in ore-forming hydrothermal systems. Geology, 17: 285-288.

Eastoe C.J., Peryt T.M., Petrychenko O.Y. \& GeislerCussey D., 2007 - Stable chlorine isotopes in Phanerozoic evaporites. Applied Geochemistry, 22(3): 575-588.

Eggenkamp H.G.M. \& Schuiling R.D., $1995-\delta^{37} \mathrm{Cl}$ variations in selected minerals: a possible tool for exploration. Journal of Geochemical Exploration, 55: 249-255.

Hawthorne F.C. \& Ferguson R.B., 1975 - Refinement of the crystal structure of kroehnkite. Acta Cryst., B31: 1753-1755.

Häuselmann A.D., Häuselmann P. \& Onac B.P., 2010 - Speleogenesis and deposition of sediments in Cioclovina Uscata Cave, Sureanu Mountains, Romania. Environmental Earth Sciences (formerly Environmental Geology), 61: 1561-1571.

Herwig S. \& Hawthorne F.C., 2006 - The topology of hydrogen bonding in brandtite, collinsite and fairfieldite. Canadian Mineralogist, 44: 1181-1196.

Hill C.A. \& Forti P., 1997 - Cave minerals of the world ( $2^{\text {nd }}$ ed.). National Speleological Society, Huntsville, Alabama, $464 \mathrm{p}$.

Kaufmann R., Long A., Bentley H. \& Davis S., 1984 - Natural chlorine isotope variations. Nature, 309: 338-340.

Kolitsch U. \& Fleck M., 2005 - Second update on compounds with kroehnkite-type chains. Z. Krist., 220: 31-41.

Kolitsch U. \& Fleck M., 2006 - Third update on compounds with kroehnkite-type chains: the crystal structure of wendwilsonite $\left(\mathrm{Ca}_{2} \mathrm{Mg}\left(\mathrm{AsO}_{4}\right)_{2} \cdot 2\left(\mathrm{H}_{2} \mathrm{O}\right)\right)$ and the new triclinic structure types of synthetic $\mathrm{AgSc}\left(\mathrm{CrO}_{4}\right)_{2} \cdot 2\left(\mathrm{H}_{2} \mathrm{O}\right)$ and $\mathrm{M}_{2}{ }_{2} \mathrm{Cu}\left(\mathrm{Cr}_{2} \mathrm{O}_{7}\right)_{2} \cdot 2\left(\mathrm{H}_{2} \mathrm{O}\right)(\mathrm{M}=$ Rb, Cs). Eur. J. Miner., 18: 471-482.

Malcherek T. \& Schlüter J., 2009 - Structures of the pseudo-trigonal polymorphs of $\mathrm{Cu}_{2}(\mathrm{OH})_{3} \mathrm{Cl}$. Acta Cryst., B65: 334-341.

Marincea Ş., Dumitraş D.-G. \& Gibert R., 2002 - Tinsleyite in the "dry" Cioclovina Cave (Şureanu Mountains, Romania): the second occurrence. Eur. J. Mineral., 14: 157-164.

Martini J.E.J., 1980 - Mbobomkulite, hydrombombomkulite and nickelalumite, new minerals from Mbobo Mkulu cave, eastern Transvaal. Ann. Geol. Surv. S. Afr., 14 (2): 1-10.

Martini J.E.J. \& Moen H.F.G., 1998 - Blue Lagoon, Afrique $d u$ Sud. Un grotte à remplissage paléokarstique permien et à concrétions d'aragonite. Karstologia, 32
(2): 27-38.

Martonne E., 1907 - L'evolution morphologique des Alpes de Transylvanie. Rev. Géogr. Annuelle, I, 278 p. Mitrofan H., 1979 - Observations sur le conditionnement tectonique du développement et de la répartition des creux du réseau Ponorici-Cioclovina cu Apa (Monts de Sebes). Trav. Inst. Spéol. "Emil Racovitza", 18: 225-231.

Nonius B.V., 1999 - "Collect". Data collection software. Brucker AXS, http://www.brucker-axs.de/.

Olariu A., Hellborg R., Stenström K., Skog G., Faarinen M., Persson P., Erlandsson B. \& Alexandrescu E., 2002 - Dating of some Romanian fossil bones by combined nuclear methods. Journal of Radioanalytical and Nuclear Chemistry, 253 (2): 307-311.

Onac B.P. \& Effenberger H., 2007 - Re-examination of berlinite $\left(\mathrm{AlPO}_{4}\right)$ from the Cioclovina Cave, Romania. American Mineralogist, 92: 1998-2001.

Onac B.P. \& Tămaş T., 2010 - Cave minerals of westcentral Romania. Acta Mineralogica-Petrographica, Field Guide Series, 22: 1-22.

Onac B.P. Effenberger H. \& Breban R.C., 2007 - Hightemperature and "exotic" minerals from the Cioclovina Cave, Romania: a review. Studia UBB Geologia, 52 (2); 3-10.

Onac B.P., Mylroie J.E. \& White W.B., 2001 - Mineralogy of cave deposits on San Salvador Island, Bahamas. Carbonates and Evaporites, 16 (1): 8-16.

Onac B.P., Breban R., Kearns J. \& Tămaş T., 2002 Unusual minerals related to phosphate deposits in Cioclovina Cave, Şureanu Mts. (Romania). Theoretical and Applied Karstology, 15: 27-34.

Onac B.P., Effenberger H., Ettinger K. \& CîntăPînzaru S., 2005a - Hydroxylellestadite from Cioclovina Cave (Romania): Microanalytical, structural, and vibrational spectroscopy data. American Mineralogist, 91: 1927-1931.

Onac B.P., Ettinger K., Kearns J. \& Balasz I.I., 2005b - A modern, guano-related occurrence of foggite, $\mathrm{CaAl}\left(\mathrm{PO}_{4}\right)(\mathrm{OH})_{2} \bullet \mathrm{H}_{2} \mathrm{O}$ and churchite- $(\mathrm{Y}), \quad \mathrm{YPO}_{4} \cdot 2 \mathrm{H}_{2} \mathrm{O}$ in Cioclovina Cave, Romania. Mineral. Petrol., 85: 291-302.

Otwinowski Z. \& Minor W., 1997 - Processing of Xray diffraction data collected in oscillation mode. In: Carter C.W.J. \& Sweet R.M. (Eds.), Methods in Enzymology, 276: 307-326.

Parise J.B. \& Hyde B.G., 1986 - The structure of atacamite and its relationships to spinel. Acta Cryst., C42: 1277-1280.

Schadler J., 1932 - Ardealit, ein neues Mineral CaH$\mathrm{PO}_{4} \mathrm{CaSO}_{4}+4 \mathrm{H}_{2} \mathrm{O}$. Centralbl. Miner. Abt. A., 40-41.

Sharp Z.D., Barnes J.D., Brearley A.J., Chaussidon M., Fischer T.P. \& Kamenetsky V.S., 2007 - Chlorine isotope homogeneity of the mantle, crust and carbonaceous chondrites. Nature, 446: 1062-1065.

Sheldrick G.M., 1997 - SHELXL-97, a program for crystal structure refinement. University of Göttingen, Germany.

Sheldrick G.M., 2008 - A short history of SHELX. Acta Cryst., A64: 112-122.

Simionescu I., 1941 - The Ursus spelaeus from the Cioclovina Cave. Analele Academiei Române, Memoriile Secțiunii Ştiințifice, seria III, 17 (7): 383-402 (in 
Romanian).

Soficaru A., Petrea C., Doboş A. \& Trinkaus E., 2007

- The human cranium from the Peştera Cioclovina Uscata, Romania. Current Anthropology, 48 (4): 611-619.

Stilla A., 1981 - Géologie de la region de HațegCioclovina-Pui-Bănița (Carpates Meridionales). Anuarul Institutului de Geologie şi Geofizică, 66: 91-179.

Tomus B., 1999 - The Ciclovina Karst Complex - Basin 2063. Clubul Speologilor Proteus, Hunedoara, 55 p. (in Romanian).

Udubaşa G. \& Hârtopanu P., 1992 - The Cioclovina occurrence: mineralogical data and some remarks on its genesis. Rom. J. Mineral Deposits, 75: 77-84.

Wilson A. J. C. (editor), 1992 - International Tables for Crystallography, Vol. C., Kluwer, Dordrecht, The Netherlands.

Wildner M. \& Stoilova D., 2003 - Crystal structures and crystal chemical relationships of kröhnkite- and collinsite-type compounds $\mathrm{Na}_{2} \mathrm{Me}^{2+}\left(\mathrm{XO}_{4}\right)_{2} \cdot 2 \mathrm{H}_{2} \mathrm{O}(\mathrm{X}=\mathrm{S}$, $M e=M n, C d ;$ and $X=S e, M e=M n, C o, N i, Z n, C d)$ and
$\mathrm{K}_{2} \mathrm{Co}\left(\mathrm{SeO}_{4}\right)_{2} \cdot 2 \mathrm{H}_{2} \mathrm{O}$. Z. Kristallogr., 218: 201-209.

Wynn J., Sumrall J. \& Onac B.P., 2010 - Sulfur isotopic composition and the source of dissolved sulfur species in thermo-mineral springs of the Cerna Valley, Romania. Chemical Geology, 271: 31-43.

Yakubovich O.V., Kabalov Yu.K., Gavrilenko P.G., Liferovich R.P. \& Massa W., 2003 - Strontium in the structure of collinsite: Rietveld method refinement. Crystallogr. Rep., 48: 226-232.

Zheng X.G., Mori T., Nishiyama K., Higemoto W., Yamada H., Nishikubo K. \& Xu C.N., 2005 - Antiferromagnetic transitions in polymorphous minerals of the natural cuprates atacamite and botallackite $\mathrm{Cu}_{2} \mathrm{Cl}(\mathrm{OH})_{3}$. Phys. Rev., Serie 3. B - Condensed Matter, 71: 174404-1-174404-8. 